\title{
Isquemia Miocárdica Intermitente após Angioplastia Cirúrgica do Tronco de Coronária Esquerda
}

\author{
Luís Roberto Gerola, Ivana Delamônica Freire Eckert, José Alfredo Sejópolis, Alberto Najjar, \\ Luís Cesar N. Scala, Ronaldo D. Fontes
}

Cuiabá, MT

\begin{abstract}
Dois pacientes do sexo masculino (38 e 53 anos) com angina instável, com estudo hemodinâmico revelando grave lesão de óstio de tronco de coronária esquerda (TCE), foram submetidos a angioplastia cirúrgica do TCE com patch de veia safena. Ao final da operação os pacientes apresentaram isquemia miocárdica intermitente, um deles com choque cardiogênico e, em ambos, reversão completa do quadro. O estudo hemodinâmico pós-operatório revelou adequada ampliação do TCE com função ventricular esquerda preservada nos dois pacientes. Embora a angioplastia cirúrgica do TCE seja uma alternativa técnica para os doentes com lesões ostiais, chamamos a atenção para esta grave morbidade operatória, tendo como uma das possíveis causas o espasmo do TCE.
\end{abstract}

\section{Intermittent Myocardial Isquemia after Surgical Angioplasty of the Left Main Coronary Artery}

Two 38 and 53-year old male patients with unstable angina, whose hemodynamic study showed an isolated stenosis of the left coronary artery ostium, were submitted to a surgical angioplasty of the left main coronary artery (LMCA) with saphenous vein patch. At the end of the operation, the patients presented intermitent myocardial isquemia. One of them led to abrupt cardiogenic shock which reversed completely. The postoperative hemodynamic study demonstrated excellent left main coronary artery patency and normal left ventricular. of LMCA has been proposed as an alternative technique to the treatment of ostial lesions, the surgical team should be prepared to face severe operative morbidity. The LMCA spasm has been considered as a possible etiology of that event.
A lesão de tronco de coronária esquerda (TCE) é fator de maior gravidade na doença coronária, sendo tratamento cirúrgico a opção terapêutica de escolha.

Aproximadamente, 5 a $9 \%$ dos pacientes com aterosclerose coronária apresentam lesão de $\mathrm{TCE}^{1,2} \mathrm{e}$ a prevalência das lesões de óstio do TCE, isolada ou não, varia de 0,1 a $1 \%{ }^{3,4}$.

Habitualmente, o tratamento cirúrgico nesta situação é realizado através da revascularização do miocárdio, utilizando-se enxertos arteriais ou venosos para as artérias interventricular anterior e circunflexa ou seus ramos.

Hospital Santa Cruz - Cuiabá

Correspondência: Luís Roberto Gerola - Rua Estado de Israel 847/ 61 - 04022 002 - São Paulo - Capital

Recebido para publicação em 27/3/97

Aceito em 23/7/97
Entretanto, nas lesões ostiais, a angioplastia cirúrgica do óstio do TCE com patch de veia safena magna é uma alternativa técnica que deve ser cogitada, principalmente em pacientes jovens, pela possibilidade de maior perviabilidade tardia, em comparação com a revascularização do miocárdio convencional, além das vantagens de se manter um fluxo coronário anterógrado ${ }^{5}$.

Relatamos dois casos de pacientes com lesão de óstio de TCE, em que, realizadas a angioplastia cirúrgica, ambos apresentaram isquemia miocárdica intermitente no intraoperatório, e um, choque cardiogênico.

\section{Relato de Caso}

Caso 1 - Homem, 38 anos, branco, natural e procedente de Cuiabá (MT). Assintomático até há uma semana quando apresentou episódio de dor precordial em aperto, em repouso, com duração de poucos minutos, sem irradiação, ces- 


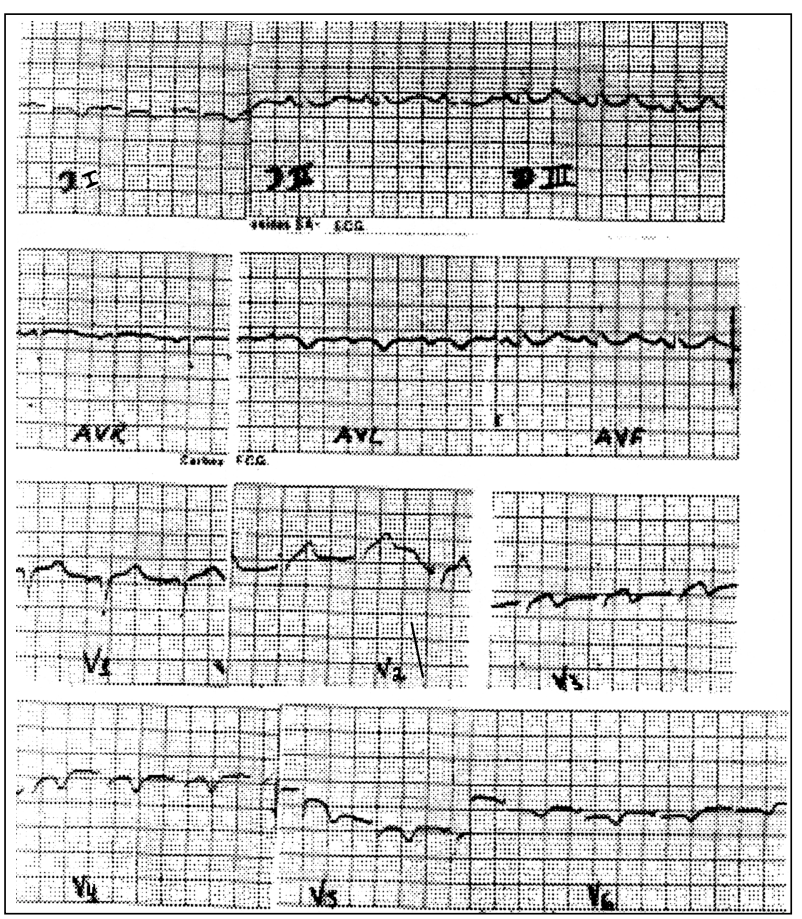

Fig. 1 - Eletrocardiograma apresentando isquemia na parede anterior e lateral-alta.

sando espontaneamente e com repetição do episódio há um dia, porém de maior intensidade. Com estes sintomas, o paciente procurou nosso serviço.

Ao exame físico estava em bom estado geral, eupnéico, acianótico, corado, hidratado, afebril, com frequiência cardíaca de 96bpm e pressão arterial (PA) de 120x 80 $\mathrm{mmHg}$. Apresentava bulhas rítmicas normofonéticas sem sopros e ausculta pulmonar normal. O abdome era plano, flácido, indolor com ruídos hidroaéreos positivos.

Oeletrocardiograma (ECG) mostrou isquemia na parede anterior e parede lateral alta (fig.1). A radiografia de tórax era normal.

Com esta história clínica e ECG, o paciente foi encaminhado para o laboratório de hemodinâmica, e realizada cinecoronariografia que revelou lesão severa de óstio e TCE (fig. 2) e ventriculografia esquerda com hipocinesia ânteroapical (fig. 3). Com este diagnóstico o paciente foi encaminhado para tratamento cirúrgico.

A operação foi realizada com circulação extracorpórea (CEC), hipotermia moderada $\left(28^{\circ} \mathrm{C}\right)$ e proteção do miocárdio realizada com cardioplegia sangüínea hipotérmica anterógrada intermitente a cada $20 \mathrm{~min}$, consistindo a técnica operatória numa aortotomia anterior, semelhante à utilizada para a troca valvar aórtica, com extensão em direção ao tronco da coronária esquerda seccionando a placa de ateroma e avançando no TCE e concluindo com a sutura do patch de veia safena com prolene 7-0, ampliando a origem do tronco.

Completada a operação, ainda em CEC e durante o aquecimento, o paciente apresentou supradesnivelamento, intermitente, do segmento ST. Optamos pela realização de um enxerto da artéria torácica interna esquerda para a artéria interventricular anterior, como medida de segurança, não havendo mais alterações eletrocardiográficas. Foi completado o aquecimento do paciente e saída de CEC sem intercorrências.

Caso 2 - Recentemente operamos outro paciente, masculino, 53 anos, com quadro clínico de angina de recente começo. O exame físico era normal, assim como os dados laboratoriais. OECG também apresentava isquemia na parede anterior e o estudo hemodinâmico revelou grave lesão isolada de óstio de TCE.

Foi realizado o mesmo procedimento cirúrgico. O paciente saiu bem de CEC e, durante o fechamento do tórax, apresentou hipotensão arterial severa (PA média de $30 \mathrm{mmHg}$ ), com grave disfunção ventricular esquerda, constatada sob visão direta do miocárdio. Realizada punção de átrio esquerdo (AE), que revelou pressão de $40 \mathrm{cmH}_{2} \mathrm{O}$. Ao monitor, oECG mostrava supradesnivelamento do segmento ST.

Iniciadas medidas de suporte com drogas vasoativas (dopamina e dobutamina) e uso de nitroglicerina endovenosa. Não havendo melhora da função ventricular, optamos por entrar novamente em CEC e realizar ponte de veia safena para a artéria interventricular anterior. Enquanto preparávamos as suturas para canulação do átrio e da aorta, houve reversão da disfunção ventricular, sendo possível a redução das drogas vasoativas, com melhora visual da contração do ventrículo esquerdo, normalização da PA média $(70 \mathrm{mmHg})$ e queda da pressão do $\mathrm{AE}\left(10 \mathrm{cmH}_{2} \mathrm{O}\right)$.

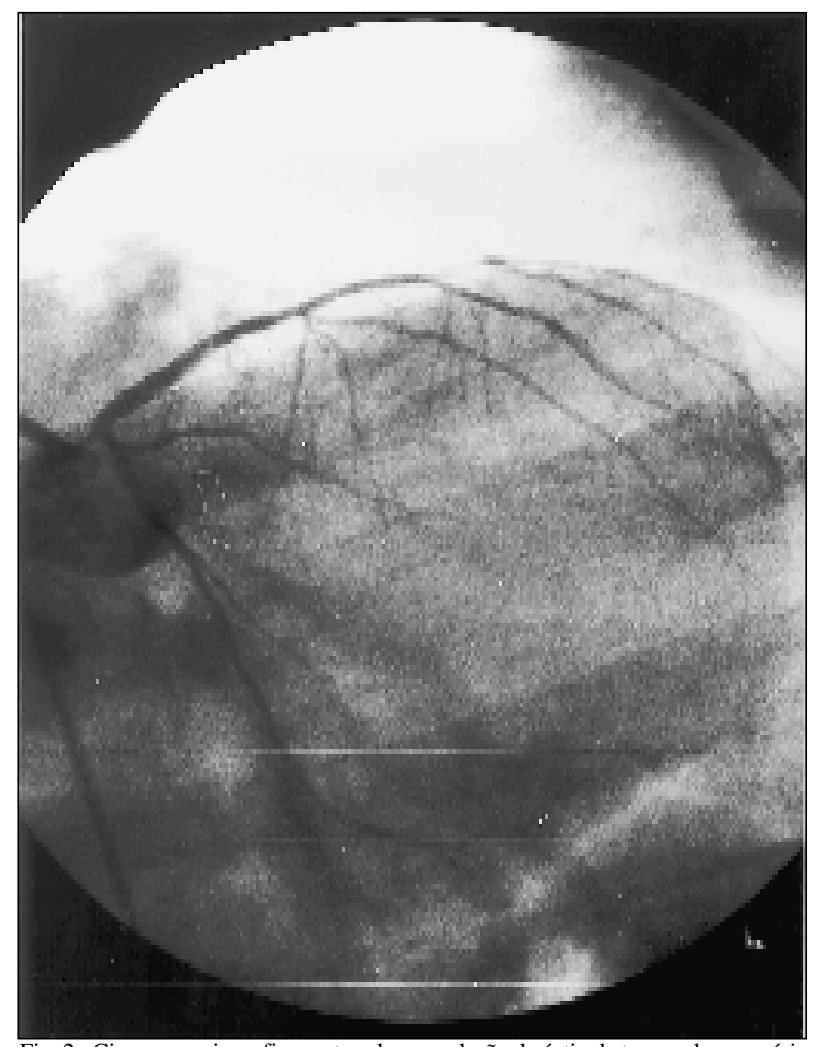

Fig. 2 - Cinecoronariografia mostrando grave lesão de óstio de tronco de coronária esquerda. 


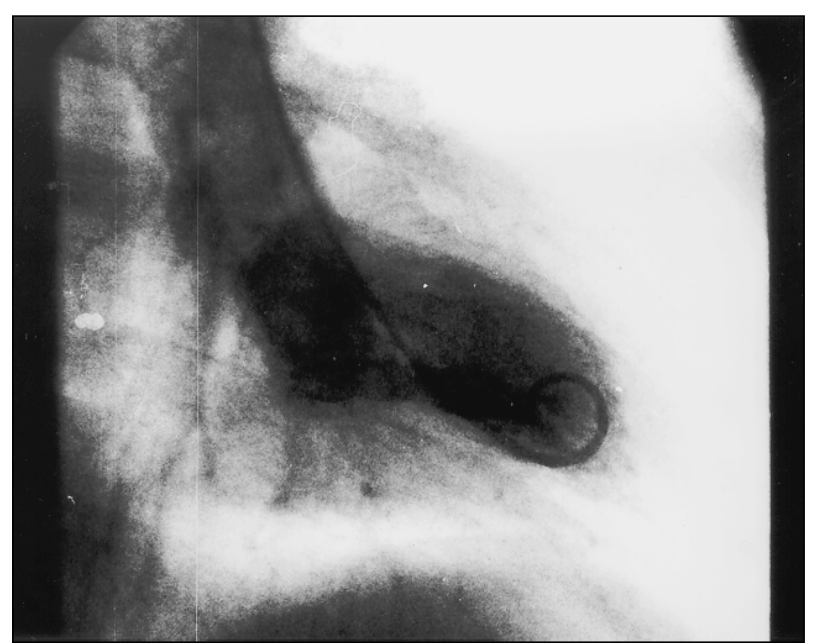

Fig. 3 - Ventriculografia esquerda pré-operatória, em sístole, apresentando hipocinesia na parede ântero-apical.

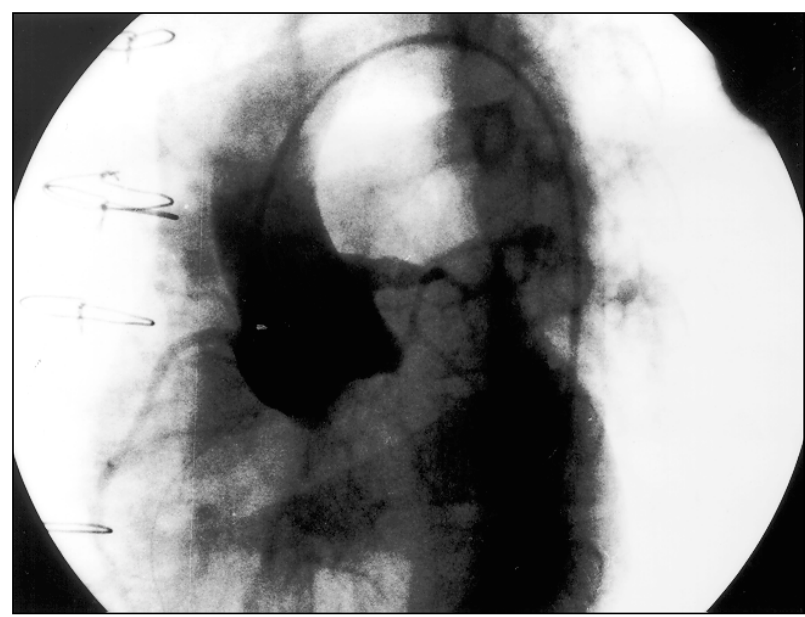

Fig. 4 - Aortografia pós-operatória onde observamos a origem do tronco de coronária esquerda com adequada ampliação.

Decidimos não realizar a ponte de safena para a artéria interventricular anterior e, após período de observação, completamos o fechamento do tórax e o paciente foi encaminhado à unidade de pós-operatório (PO).

No PO, o ECG era normal, semelhante ao pré-operatório, sem alterações enzimáticas. O paciente evoluiu com disfunção neurológica transitória caracterizada por sonolência, agitação psicomotora e hemiparesia à esquerda, permanecendo entubado por $48 \mathrm{~h}$. Após este período foi extubado, evoluindo com reversão completa do déficit neurológico.

No $5^{\circ}$ dia de PO foi realizado estudo hemodinâmico em ambos os pacientes, que mostrou adequada ampliação do TCE (fig. 4 e 5), sem lesões, bom fluxo pela artéria coronária esquerda e com função ventricular preservada (fig. 6).

No paciente em que foi utilizada a artéria torácica interna esquerda, esta apresentava-se pérvia, porém com competição de fluxo e afilada.

Os pacientes receberam alta no $6^{\circ}$ dia de $\mathrm{PO}$, em classe funcional I (NYHA), medicados com ácido acetil salicílico $100 \mathrm{mg} /$ dia.

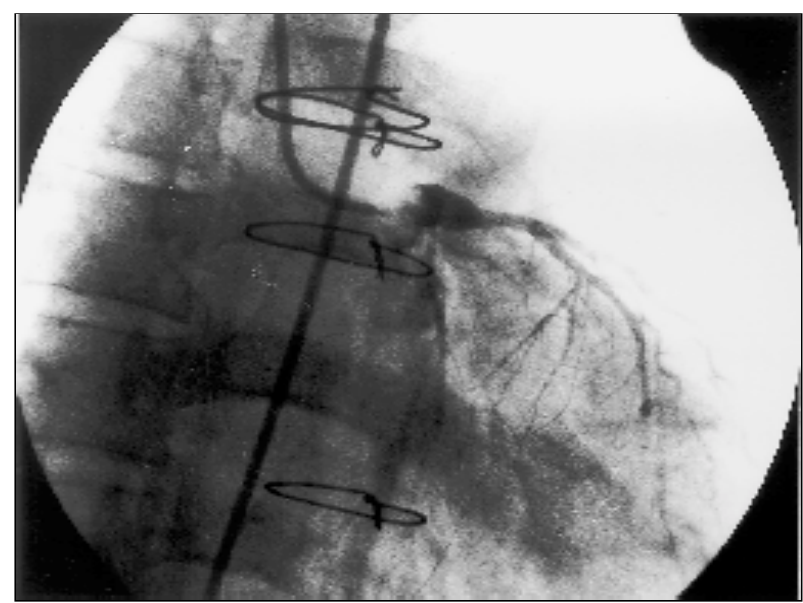

Fig. 5 - Coronariografia seletiva do tronco de coronária esquerda demonstrando a ampliação com enchimento distal das artérias coronárias.

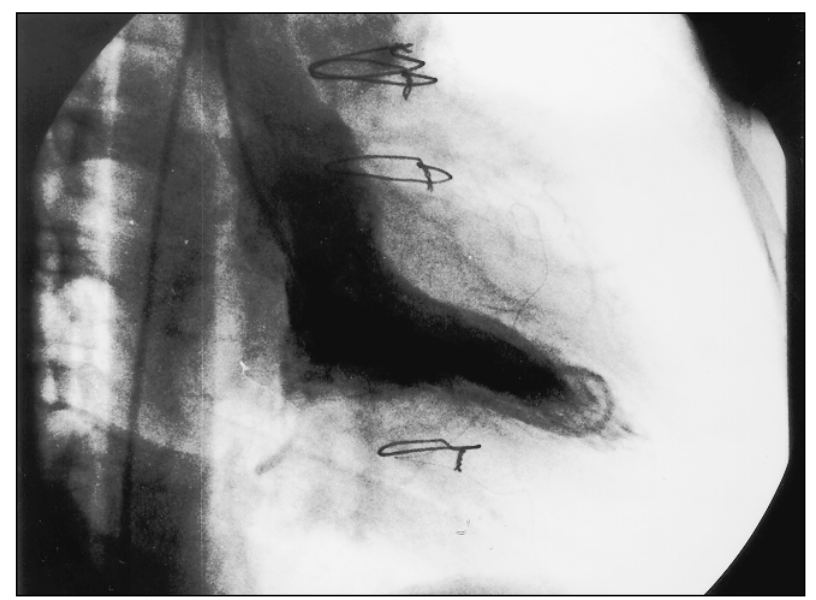

Fig. 6 - Ventriculografia esquerda pós-operatória, em sístole, com melhora da função ventricular esquerda, em relação ao pré-operatório.

\section{Discussão}

As primeiras tentativas de angioplastia cirúrgica do TCE com patch de veia safena foram descritas por Effer e $\mathrm{col}^{6}$ e por Sabiston e col ${ }^{7} \mathrm{em} 1965$. No entanto, este procedimento foi abandonado devido à elevada mortalidade, maior que $45 \%$.

Em 1983, Hithcock e col ${ }^{8}$ reviveram esta técnica apresentando nove pacientes operados, sem óbitos ou infarto Pós Operatório, com boa evolução tardia, num período médio de seguimento de 1,9 anos. Atribuem os bons resultados à melhoria das técnicas de proteção do miocárdio e aos melhores recursos no intra e PO.

Em nosso meio, Carvalho e col ${ }^{9}$, em 1984, fizeram o $1^{\circ}$ relato de caso da literatura nacional sobre esta técnica, com excelente resultado.

Com a divulgação da técnica, outros autores apresentaram sua experiência e constatamos que a isquemia miocárdica, com ou sem disfunção ventricular, foi observada no PO.

Sullivan e col ${ }^{10}$ estudaram 14 pacientes com lesão de óstio de TCE tratados com angioplastia cirúrgica sem óbi- 
tos. Comentam que dois pacientes apresentaram supradesnivelamento intermitente do segmento ST no PO, sendo que um com baixo débito grave, melhorado progressivamente com uso de nitroglicerina endovenosa e drogas vasoativas. O estudo hemodinâmico PO demonstrou adequada ampliação do TCE. Os autores sugerem a possibilidade de espasmo como responsável pelas alterações ocorridas.

Dion e col ${ }^{11}$ relatam 23 casos operados utilizando a angioplastia cirúrgica do TCE com baixa mortalidade e boa evolução tardia (24,3 meses). Entretanto, quatro pacientes apresentaram choque cardiogênico súbito durante o fechamento do tórax, sendo necessária a revascularização do miocárdio com pontes de veia safena para as artérias descendente anterior e circunflexa.

Há vantagens da angioplastia cirúrgica sobre a revascularização do miocárdio convencional? A angioplastia cirúrgica do TCEé um procedimento de maior complexidade técnica, além disto, os relatos da literatura são recentes e não apresentam análises comparativas com relação à perviabilidade tardia, entre essa técnica e a revascularização convencional.

No presente estudo, relatamos dois pacientes com lesão de TCE em que foi realizada a angioplastia cirúrgica e ambos apresentaram isquemia miocárdica súbita, um deles com choque cardiogênico.

Esta característica paroxística, com reversão completa e espontânea, torna o espasmo do TCE uma hipótese pro- vável. O estímulo direto do TCE, ou a presença de hematomas, causados pela dissecção, infiltrando as camadas da artéria, poderiam desencadear este espasmo. Com o fim do espasmo e o tronco ampliado, o fluxo sangüíneo para o VE normalizar-se-ia justificando a recuperação da função ventricular.

No paciente em que foi realizado enxerto com a artéria torácica interna esquerda, o aumento de fluxo através da circulação coronária causaria um fluxo competitivo, razão pela qual a artéria torácica interna esquerda encontrava-se afilada e com calibre reduzido no estudo hemodinâmico PO.

Os nossos resultados, à semelhança de Dion e col ${ }^{11} \mathrm{e}$ Sullivan e col $^{10}$, demonstraram a possibilidade da disfunção do miocárdio ser um risco sempre presente, podendo ter sido o responsável pela elevada mortalidade inicial descrita por Effer e col ${ }^{6}$.

O uso de nitroglicerina endovenosa durante o aquecimento do paciente, ainda em CEC, além de bloqueadores de canal de cálcio, talvez possa evitar ou diminuir a ocorrência desta complicação.

Em conclusão, acreditamos que esta técnica deve ser realizada em grupos selecionados de pacientes, com idade $<60$ anos, lesão isolada de óstio de TCE e função ventricular preservada. A possibilidade de ocorrer isquemia miocárdica intermitente deve ser sempre lembrada, estando a equipe cirúrgica preparada para esta grave complicação operatória.

\section{Referências}

1. Farinka JB, Kapaln MA, Harris CN et al - Disease of left main coronary artery. Surgical treatment and long term follow-up in 267 patients. Am J Cardiol 1978; 42: $124-8$.

2. De Mots H, Rösch J, Mc Anulty JH - Left main coronary artery disease. Cardiovasc Clin 1977; 8: 201-11.

3. Hunter JÁ, Pasaoglu I, Willians BT - The incidence and management of coronary ostial stenosis. J Cardiovasc Surg 1985; 26: 581-4.

4. Barner HB, Naunheim KS, Kanter KR et al - Coronary ostial stenosis. Eur J Thorac Cardiovasc Surg 1988; 2: 106-12.

5. Evans CL, Hartridge H - Dynamics of the Circulation, Principles of Human Physiology. London: Churchill Livingstone, 1952.

6. Effer DB, Sones FM, Favaloro R, Groves LK - Coronary endarterectomy with patch graft reconstruction. Clinical experience with 34 cases. Ann Surg 1965; 162: 590-601.
7. Sabiston DC, Ebert PA, Friesinger GC, Ross RS, Sinclair-Smith B - Proximal endarterectomy artery reconstruction for coronary occlusion at aortic origin. Arch Surg 1965; 91: 758-64.

8. Hitchcock JF, Robles de Medina EO, Jambroes G - Angioplasty of the left main coronary artery for isolated left main coronary artery disease. J Thorac Cardiovasc Surg 1983; 85: 880-4.

9. Carvalho RG, Ribeiro EJ, Brofman PR, Rocha Loures D - Angioplastia cirúrgica do óstio da artéria coronária esquerda. Arq Bras Cardiol 1984; 42: 355-69.

10. Sullivan JÁ, Murphy DA-Surgical repair of stenotic ostial lesions of the left main coronary artery. J Thorac Cardiovasc Surg 1989; 98: 33-6.

11. Dion R, Verhelst R, Matta A, Rousseau M, Goenen M, Chalant C-Surgical angioplasty of the left main coronary artery. J Thorac Cardiovasc Surg 1990; 99: 241-50. 